\title{
Generating relativistic pseudo-potentials with explicit incorporation of semi-core states using APE, the Atomic Pseudo-potentials Engine
}

\author{
Micael J.T. Oliveira*, Fernando Nogueira \\ Centre of Computational Physics, University of Coimbra, Rua Larga, 3004-516 Coimbra, Portugal \\ Received 11 September 2007; accepted 8 November 2007 \\ Available online 26 December 2007
}

\begin{abstract}
We present a computer package designed to generate and test norm-conserving pseudo-potentials within Density Functional Theory. The generated pseudo-potentials can be either non-relativistic, scalar relativistic or fully relativistic and can explicitly include semi-core states. A wide range of exchange-correlation functionals is included.
\end{abstract}

\section{Program summary}

Program title: Atomic Pseudo-potentials Engine (APE)

Catalogue identifier: AEAC_v1_0

Program summary URL: http://cpc.cs.qub.ac.uk/summaries/AEAC_v1_0.html

Program obtainable from: CPC Program Library, Queen's University, Belfast, N. Ireland

Licensing provisions: Standard CPC licence, http://cpc.cs.qub.ac.uk/licence/licence.html

No. of lines in distributed program, including test data, etc.: 88287

No. of bytes in distributed program, including test data, etc.: 649959

Distribution format: tar.gz

Programming language: Fortran 90, C

Computer: any computer architecture, running any flavor of UNIX

Operating system: GNU/Linux

RAM: $<5 \mathrm{Mb}$

Classification: 7.3

External routines: GSL (http://www.gnu.org/software/gs1/)

Nature of problem: Determination of atomic eigenvalues and wave-functions using relativistic and nonrelativistic Density-Functional Theory. Construction of pseudo-potentials for use in ab-initio simulations.

Solution method: Grid-based integration of the Kohn-Sham equations.

Restrictions: Relativistic spin-polarized calculations are not possible. The set of exchange-correlation functionals implemented in the code does not include orbital-dependent functionals.

Unusual features: The program creates pseudo-potential files suitable for the most widely used ab-initio packages and, besides the standard non-relativistic Hamann and Troullier-Martins potentials, it can generate pseudo-potentials using the relativistic and semi-core extensions to the Troullier-Martins scheme. APE also has a very sophisticated and user-friendly input system.

Running time: The example given in this paper ( $\mathrm{Si}$ ) takes $10 \mathrm{~s}$ to run on a Pentium IV machine clocked at $2 \mathrm{GHz}$.

(c) 2007 Elsevier B.V. All rights reserved.

\footnotetext{
th This paper and its associated computer program are available via the Computer Physics Communications homepage on ScienceDirect (http://www.sciencedirect. com/science/journal/00104655).

* Corresponding author.

E-mail address: micael@teor.fis.uc.pt (M.J.T. Oliveira).
} 


\section{Introduction}

Density-functional theory [1] (DFT) methods have become the methods of choice to study a wide range of phenomena. At the base of this success is the affordable computational effort they require and one of the key ingredients contributing to this is the pseudo-potential approximation.

In the pseudo-potential approximation the electrons in the atom are separated into valence and inner core electrons. Since the inner core electrons are strongly bound they do not play a significant role in the chemical binding of atoms. This means they can safely be ignored in a large number of cases and the potential felt by the valence electrons can be replaced by an effective interaction that accounts for the nuclear attraction and all the interactions between the valence and the inert inner core electrons. This effective interaction is the pseudo-potential.

Many different schemes have been proposed to generate pseudo-potentials [2-7]. In recent years some new developments occurred in pseudo-potential theory: two extensions of the Troullier-Martins scheme were proposed, one to include semi-core states explicitly [8] and the other to obtain pseudopotentials from a fully relativistic all-electron calculation in a consistent way [9].

In this article we present a computer package to generate and test norm-conserving pseudo-potentials that include these novel features. We wrote the code in Fortran 90/95 using modern programming concepts so that it should be easy to add new features.

The program can output the pseudo-potentials in four different formats, corresponding essentially to four different widely used ab-initio packages: SIESTA [10], FHI98PP [11], ABINIT [12], and PWscf [13].

\section{Density Functional Theory for atoms}

In this section we will take a brief overlook on density functional theory for atoms, both relativistic and non-relativistic, and/or the numerical details of our implementation.

\subsection{Non-relativistic equations}

Within non-relativistic Kohn-Sham DFT [14], the ground state of a system of $N$ electrons subject to an external potential can be obtained by solving a set of one-particle equations, the Kohn-Sham equations [14] (atomic units will be used throughout):

$$
\begin{aligned}
& {\left[-\frac{\nabla^{2}}{2}+v_{\mathrm{KS}}[n](\vec{r})\right] \varphi_{i}(\vec{r})=\varepsilon_{i} \varphi_{i}(\vec{r}),} \\
& n(\vec{r})=\sum_{i}^{\text {occ }}\left|\varphi_{i}(\vec{r})\right|^{2} .
\end{aligned}
$$

The Kohn-Sham potential $v_{\mathrm{KS}}[n](\vec{r})$, a functional of the electronic density $n(\vec{r})$, is usually divided as:

$v_{\mathrm{KS}}[n](\vec{r})=v_{\mathrm{ext}}(\vec{r})+v_{\mathrm{H}}[n](\vec{r})+v_{\mathrm{xc}}[n](\vec{r})$,

where $v_{\text {ext }}$ is the external potential, $v_{\mathrm{H}}$ is the Hartree potential and $v_{\mathrm{xc}}$ is the exchange and correlation potential. For simplicity we will from now on omit the electronic density when referring to the Kohn-Sham potential: $v_{\mathrm{KS}}(\vec{r}) \equiv v_{\mathrm{KS}}[n](\vec{r})$.

In the case of an isolated atom, $v_{\mathrm{ext}}(\vec{r})$ is just the nuclear $-\frac{Z}{r}$ potential. Taking into account the spherical symmetry of the problem, one normally performs a spherical averaging of the density. This leads to a spherically symmetric Kohn-Sham potential and thus the Kohn-Sham orbitals can be separated into angular and radial parts:

$\varphi_{i}(\vec{r})=R_{n l}(r) Y_{l m}(\theta, \phi)$.

$Y_{l m}(\theta, \phi)$ are spherical harmonics and $R_{n l}(r)$ are the solutions of a "simple" one-dimensional second-order differential equation:

$\left[-\frac{1}{2} \frac{\mathrm{d}^{2}}{\mathrm{~d} r^{2}}-\frac{1}{r} \frac{\mathrm{d}}{\mathrm{d} r}+\frac{l(l+1)}{2 r^{2}}+v_{\mathrm{KS}}(r)\right] R_{n l}(r)=\varepsilon_{n l} R_{n l}(r)$.

Because of the spherical symmetry of the system, states with the same $n$ and $l$ quantum numbers are degenerate and therefore equally occupied. As a consequence, the radial electron density can be written as:

$n(r)=\sum_{n} \sum_{l=0}^{n-1} \Theta_{n l} \frac{\left|R_{n l}(r)\right|^{2}}{4 \pi}$,

with $\Theta_{n l}$ denoting the occupation of each $n l$ sub-shell.

When dealing with atoms with net spin it is necessary to use spin density functional theory [15]. In that case the exchangecorrelation potential depends not only on the density $n(r)=$ $n(r, \sigma=\uparrow)+n(r, \sigma=\downarrow)$ but also on the magnetization density $m(r)=n(r, \sigma=\uparrow)-n(r, \sigma=\downarrow)$ and the spin-up and spin-down electrons feel a different Kohn-Sham potential. This means there are two sets of one-particle equations to be solved.

\subsection{Relativistic equations}

When relativistic effects become important one needs to incorporate them into the Kohn-Sham equations. One possible way is to use the scalar-relativistic scheme of Koelling and Harmon [16] which omits the spin-orbit interaction but includes all other relativistic kinematic effects. In that case the $R_{n l}(r)$ are the solutions of the following one-dimensional second-order differential equation:

$$
\begin{aligned}
& \frac{1}{2 M(r)}\left(-\frac{\mathrm{d}^{2}}{\mathrm{~d} r^{2}}+\frac{1}{M(r)} \frac{\mathrm{d} M(r)}{\mathrm{d} r} \frac{\mathrm{d}}{\mathrm{d} r}-\frac{2}{r} \frac{\mathrm{d}}{\mathrm{d} r}+\frac{l(l+1)}{r^{2}}\right) R_{n l}(r) \\
& \quad+v_{\mathrm{KS}}(r) R_{n l}(r)=\varepsilon_{n l} R_{n l}(r),
\end{aligned}
$$


with the relativistic electron mass $M(r)$ given by $M(r)=1+$ $\left(\varepsilon_{n l}-v_{\mathrm{KS}}(r)\right) / 2 c^{2}$.

Another way to incorporate relativistic effects is to use the relativistic extension of DFT [17] (RDFT). In RDFT the KohnSham equations are replaced by the Dirac-Kohn-Sham equations:

$\left[-i c \vec{\alpha} \cdot \vec{\nabla}+\beta c^{2}+v_{\mathrm{KS}}(\vec{r})\right] \varphi_{i}(\vec{r})=\varepsilon_{i} \varphi_{i}(\vec{r})$,

$n(\vec{r})=\sum_{i}^{\text {occ }} \varphi_{i}^{\dagger}(\vec{r}) \varphi_{i}(\vec{r})$.

In a similar way as for the non-relativistic case, if $v_{\text {ext }}$ is just the nuclear $-\frac{Z}{r}$ potential then the Dirac-Kohn-Sham spinors $\varphi_{i}(\vec{r})$ can be separated into angular and radial parts:

$\varphi_{i}(\vec{r})=\left(\begin{array}{c}i g_{n l j}(r) \Omega_{j l m}(\theta, \phi) \\ -f_{n l j}(r) \Omega_{j l^{\prime} m}(\theta, \phi)\end{array}\right)$.

$\Omega_{j l m}(\theta, \phi)$ and $\Omega_{j l^{\prime} m}(\theta, \phi)$ are the spherical spinors and $g_{n l j}(r)$ and $f_{n l j}(r)$ are the solutions of a set of two coupled first-order differential equations (with $\varepsilon_{i} \rightarrow \varepsilon_{n l j}+c^{2}$ ):

$$
\begin{gathered}
c\left[\frac{\mathrm{d} g_{n l j}(r)}{\mathrm{d} r}+\frac{1+k}{r} g_{n l j}(r)\right] \\
-\left[\varepsilon_{n l j}+2 c^{2}-v_{\mathrm{KS}}(r)\right] f_{n l j}(r)=0, \\
c\left[\frac{\mathrm{d} f_{n l j}(r)}{\mathrm{d} r}+\frac{1-k}{r} f_{n l j}(r)\right] \\
\quad+\left[\varepsilon_{n l j}-v_{\mathrm{KS}}(r)\right] g_{n l j}(r)=0 .
\end{gathered}
$$

The quantum numbers $k, l$ and $l^{\prime}$ are related to each other in the following way:

$l^{\prime}=l-k /|k|$,

$k= \begin{cases}-(l+1) & \text { for } j=l+\frac{1}{2}, \\ l & \text { for } j=l-\frac{1}{2} .\end{cases}$

Taking into account the degeneracy of states with the same $n$, $j$, and $l$ quantum numbers the radial electron density can be written as:

$n(r)=\sum_{n} \sum_{l=0}^{n-1} \sum_{j=l-\frac{1}{2}}^{l+\frac{1}{2}} \Theta_{n l j} \frac{\left|f_{n l j}(r)\right|^{2}+\left|g_{n l j}(r)\right|^{2}}{4 \pi}$.

\subsection{Numerical details}

\subsubsection{Solution of the single-particle equations}

Eqs. (9) for the fully relativistic case are of first order. Second-order equations (4) and (6) for the non-relativistic and scalar-relativistic cases can be trivially transformed into a set of coupled first-order equations. Then, whatever formulation of DFT used, one is left with two coupled one-dimensional firstorder differential equations and the same numerical method can be used to solve them. One should note that the eigenvalues $\varepsilon_{n l}$ must be obtained simultaneously with the eigenfunctions.

The solutions of the single-particle equations, for a given, fixed, electronic density, are obtained in the following way:
(1) Let $f(r)$ and $g(r)$ be the functions to be determined when solving the set of differential equations.

(2) For a given energy $\varepsilon_{n l}$ choose two arbitrary values $f\left(r_{0}\right)$ and $f\left(r_{\infty}\right)$ where $r_{0}$ is a point close to the origin and $r_{\infty}$ is a point very far away.

(3) Compute $g\left(r_{0}\right)$ and $g\left(r_{\infty}\right)$ using boundary conditions determined from the equations to solve when $r \rightarrow 0$ and $r \rightarrow \infty$.

(4) Knowing $f\left(r_{0}\right)$ and $g\left(r_{0}\right)$ and using a suitable algorithm to solve ordinary differential equations it is possible to obtain the values of $f$ and $g$ at a point $r_{m}$ lying between $r_{0}$ and $r_{\infty}$. A good choice for $r_{m}$ is the classical turning point.

(5) Repeat the previous step, but starting the integration from "practical infinity" $r_{\infty}$.

(6) Let $f_{1}(r)$ and $g_{1}(r)$ be the functions obtained at step (3) and $f_{2}(r)$ and $g_{2}(r)$ the functions obtained at step (4). Multiply $f_{1}(r)$ and $g_{1}(r)$ by $f_{2}\left(r_{m}\right) / f_{1}\left(r_{m}\right)$. This makes $f(r)$ continuous at $r_{m}$.

(7) $\varepsilon_{n l}$ is an eigenvalue when $g_{1}\left(r_{m}\right)=g_{2}\left(r_{m}\right)$, so the problem of finding the eigenvalues can be reduced to the problem of finding the roots of the function $g_{1}\left(r_{m}\right)-g_{2}\left(r_{m}\right)$.

There are several methods that can be used at steps (4) and (5). We have chosen to rely on the methods available in the GNU Scientific Library [18] (GSL) that provide the solutions on an adaptive grid. As for the root finding problem (step (7)), since it is easy enough to bracket the eigenvalues, APE uses Brent's method [19] to find the roots within a given tolerance.

\subsubsection{Self-consistent cycle}

The eigenfunctions obtained with the procedure outlined in the previous section are used to rebuild the electronic density (Eqs. (5) or (11)). As the Kohn-Sham potential depends on the density, a new density corresponds to a new potential, and the differential equations have to be solved again, in a selfconsistent manner. The self-consistency cycle ends when some stopping criterion is met. In APE, the stopping criterion is based on the difference between the new and the old Kohn-Sham potential.

\subsubsection{Grids}

All the quantities that need to be kept in memory are stored on a radial logarithmic grid. This is the most suitable kind of grid for this purpose since a higher density of points is needed near the origin. Unfortunately there is more than one way to define a logarithmic grid, so we implemented two commonly used ways to construct it. In both cases, two parameters $a$ and $b$ are required. The $i$ th point of the grid is defined as $r_{i}=b e^{a i}$ in one case and as $r_{i}=b e^{a(i-1)}$ in the other case. The parameters $a$ and $b$ are determined by specifying the number of grid points and the starting and ending points.

Since some of the quantities are determined on an adaptive grid, cubic splines are used to extrapolate their values on the logarithmic grid. The numerical calculation of integrals and derivatives is also done using cubic splines. All the spline operations are handled by the GSL. 


\subsubsection{Hartree and exchange-correlation potential}

The evaluation of the Hartree potential is straightforward in the case of a spherically symmetric density:

$v_{\text {Hartree }}(r)=\frac{4 \pi}{r} \int_{0}^{r} \mathrm{~d} r^{\prime} r^{\prime 2} n\left(r^{\prime}\right)+4 \pi \int_{r}^{\infty} \mathrm{d} r^{\prime} r^{\prime} n\left(r^{\prime}\right)$.

For the evaluation of the exchange and correlation potential, in order to provide as many functionals as possible, we use a library of functionals written in $\mathrm{C}$ for the Octopus project [20]. This library provides a great number of LDA and GGA functionals as well as their relativistic extensions.

\subsubsection{Mixing}

In order to avoid instabilities during the self-consistent cycle, the input density for the next iteration is built using the densities of the previous iterations. Both linear mixing and the generalized Broyden algorithm of Johnson [21] are implemented in the code.

\section{Pseudo-potential generation and testing}

In this section we will briefly introduce norm-conserving pseudo-potentials and the different schemes implemented in APE to generate them. We will then present the KleinmanBylander form of the pseudo-potentials and finalize with some considerations on pseudo-potential transferability.

\subsection{Norm-conserving pseudo-potentials}

Modern pseudo-potentials are obtained from all-electron atomic calculations and are able to describe accurately the chemically relevant part of the valence wave-functions and charge density [22]. The procedure to obtain many of them, the so-called norm-conserving pseudo-potentials, is the following:

(1) The radial Kohn-Sham equations for the free atom are solved, using the methods expounded in the previous section, for a given reference configuration. We thus obtain the all-electron wave-functions $R_{l}^{\mathrm{AE}}(r)$ and potential $v_{\mathrm{KS}}^{\mathrm{AE}}$.

(2) The pseudo wave-functions $R_{l}^{\mathrm{PP}}(r)$ are determined using a suitable scheme that obeys several generic conditions.

(3) The screened pseudo-potential $v_{l}^{\mathrm{PP}, \mathrm{scr}}(r)$ results from the inversion of a radial Schrödinger-like Kohn-Sham equation for the pseudo wave-function and the valence electronic density

$v_{l}^{\mathrm{PP}, \mathrm{scr}}(r)=\epsilon_{l}^{\mathrm{PP}}-\frac{l(l+1)}{2 r^{2}}+\frac{1}{2 r R_{l}^{\mathrm{PP}}(r)} \frac{\mathrm{d}^{2}}{\mathrm{~d} r^{2}} r R_{l}^{\mathrm{PP}}(r)$.

(4) The Hartree and the exchange-correlation screening contributions from the valence electrons are subtracted to the screened pseudo-potentials in order to obtain the final pseudo-potentials

$$
v_{l}^{\mathrm{PP}}(r)=v_{l}^{\mathrm{PP}, \mathrm{scr}}(r)-v_{\text {Hartree }}\left[n^{\mathrm{PP}}\right](r)-v_{\mathrm{xc}}\left[n^{\mathrm{PP}}\right](r) .
$$

The conditions imposed on the pseudo wave-functions are the following:

(1) The pseudo wave-functions coincide with the all-electron valence wave-functions beyond a certain radius $r_{l}$ known as the core cutoff radius

$$
R_{l}^{\mathrm{PP}}(r)=R_{n l}^{\mathrm{AE}}(r), \quad \text { if } r>r_{l} .
$$

Also, the pseudo wave-functions are normalized. These conditions impose the following norm-conservation constraint:

$$
\int_{0}^{r^{\prime}} \mathrm{d} r\left|R_{l}^{\mathrm{PP}}(r)\right|^{2} r^{2}=\int_{0}^{r^{\prime}} \mathrm{d} r\left|R_{n l}^{\mathrm{AE}}(r)\right|^{2} r^{2}, \quad \text { if } r^{\prime} \geqslant r_{l} .
$$

(2) The pseudo energy-eigenvalues match the all-electron eigenvalues

$$
\epsilon_{l}^{\mathrm{PP}} \equiv \epsilon_{n l}^{\mathrm{AE}} .
$$

(3) The pseudo wave-functions should not have nodal surfaces.

As a consequence of the previous conditions it comes that the logarithmic derivatives of the pseudo wave-functions agree with the all-electron wave-functions beyond the core cutoff radius:

$\frac{\mathrm{d}}{\mathrm{d} r} \ln R_{l}^{\mathrm{PP}}(r)=\frac{\mathrm{d}}{\mathrm{d} r} \ln R_{l}^{\mathrm{AE}}(r)$.

As it can be seen above, the norm-conserving pseudo-potentials are spherically symmetric and $l$-dependent. Because of that, the pseudo-potential operator is non-local in the angular variables and local in the radial variable and it can be written in the following semi-local form:

$\hat{v}_{\mathrm{SL}}=\sum_{l m}\left|Y_{l m}\right\rangle v_{l}^{\mathrm{PP}}(r)\left\langle Y_{l m}\right|$.

Up to here we assumed the wave-functions and wave-equations to be non-relativistic. Nevertheless it is possible to include relativistic effects in the pseudo-potentials. To do that one starts from a relativistic all-electron calculation and generates the pseudo-potentials for both $j=l+1 / 2$ and $j=l-1 / 2$. One may use directly these $j$-dependent pseudo-potentials, but it may be convenient to remove this dependency by separating the scalar-relativistic effects from the spin-orbit coupling term. This is done by defining:

$v_{l}^{\mathrm{Avg}}=\frac{l}{2 l+1}\left[(l+1) v_{l+1 / 2}^{\mathrm{PP}}+l v_{l-1 / 2}^{\mathrm{PP}}\right]$,

$\delta v_{l}^{\mathrm{SO}}=\frac{1}{2 l+1}\left[v_{l+1 / 2}^{\mathrm{PP}}-v_{l-1 / 2}^{\mathrm{PP}}\right]$.

$v_{l}^{\mathrm{Avg}}$ can be used directly in Eq. (19), thus neglecting the spinorbit coupling term. When including spin-orbit effects Eq. (19) becomes:

$\hat{v}_{\mathrm{SL}}=\sum_{l m}\left|Y_{l m}\right\rangle\left[v_{l}^{\mathrm{Avg}}(r)+\delta v_{l}^{\mathrm{SO}} \mathbf{L} \cdot \mathbf{S}\right]\left\langle Y_{l m}\right|$. 


\subsection{Spin polarization}

If the atomic reference configuration is spin-polarized, the resulting pseudo-potentials will be spin-dependent. But the pseudo-potentials represent the effect of the inert ionic core. Therefore, this spin-dependence can be interpreted as a net magnetization of the core. However, in most cases, spin polarization of the core is negligible. The most usual way to deal with this side-effect is to perform a spin-averaging of the pseudopotential:

$v_{l}^{\mathrm{SA}}=\frac{N_{e}^{\uparrow}}{N_{e}} v_{l}^{\uparrow}+\frac{N_{e}^{\downarrow}}{N_{e}} v_{l}^{\downarrow}$

( $N_{e}$ denotes the total number of valence electrons and $N_{e}^{\uparrow}$ and $N_{e}^{\downarrow}$ represent the number of electrons with spin up and down, respectively). This spin-averaging is in fact an approximation, as we assume that all spin-polarization comes from the valence electrons. The approximation breaks down for, e.g., transitionmetal atoms like $\mathrm{Fe}, \mathrm{Ni}$, and $\mathrm{Cr}$. In these cases one can recover core polarization by explicitly including semi-core states in the pseudo-potential or by the generation of a perturbative correction to the spin-independent pseudo-potential [23]. In the latter case, the spin-dependent potential is constructed only at the moment of its application, as the local magnetization density is required. APE only performs the spin-averaging, dealing with core polarization through the MRPP scheme (see below).

\subsection{Unbound states}

In order to generate the pseudo-potential for channels associated to unbound states, the code offers two options for the pseudo-state reference energy. The first option is the one used in José Luís Martins' code [24], i.e., the reference energy is assumed to be zero. The other option is the one used in the FHI98PP code [11] which consists in considering that all pseudo-states corresponding to unbound all-electron levels have the same energy as the least bound state.

\subsection{Pseudo-potential generation schemes}

\subsubsection{Hamann potential}

One of the most used pseudo wave-functions parametrizations is the one proposed in 1979 by Hamann, Schlüter, and Chiang [25] and later improved by Bachelet, Hamann and Schlüter [26] and Hamann [3].

The method they proposed consists in using an intermediate pseudo-potential, $\bar{v}_{l}^{\mathrm{PP}}(r)$, given by

$\bar{v}_{l}^{\mathrm{PP}}(r)=v_{\mathrm{KS}}^{\mathrm{AE}}\left[n^{\mathrm{AE}}\right](r)\left[1-f\left(\frac{r}{r_{l}}\right)\right]+c_{l} f\left(\frac{r}{r_{l}}\right)$,

where $f(x)=\mathrm{e}^{-x^{\lambda}}$, and $\lambda=4.0[25]$ or $\lambda=3.5[3,26]$.

The Kohn-Sham equations are solved using this intermediate pseudo-potential, and the constants $c_{l}$ are adjusted in order to obey (17). This results in a set of intermediate wave-functions $\bar{R}_{l}(r)$. Notice that the form of the wave-functions implies that norm-conservation (Eq. (16)) is verified for some $\tilde{r}_{l}>r_{l}$.
As the two effective potentials are identical for $r>\tilde{r}_{l}$, and given the fast decay of $f(x)$, the intermediate pseudo wave-functions coincide, up to a constant, with the true wave-functions in that region.

In the method proposed by Hamann [3], the parameters $c_{l}$ are adjusted so that

$\left.\frac{\mathrm{d}}{\mathrm{d} r} \ln \left[r R_{n l}^{\mathrm{AE}}(r)\right]\right|_{r=\tilde{r}_{l}}=\left.\frac{\mathrm{d}}{\mathrm{d} r} \ln \left[r \bar{R}_{l}(r)\right]\right|_{r=\tilde{r}_{l}}$.

This way, the method is not restricted to bound states.

To impose norm-conservation (Eq. (16)), the final pseudo wave-functions, $R_{l}^{\mathrm{PP}}(r)$, are defined as a correction to the intermediate wave-functions

$R_{l}^{\mathrm{PP}}(r)=\gamma_{l}\left[\bar{R}_{l}(r)+\delta_{l} g_{l}(r)\right]$,

where $\gamma_{l}$ is the ratio $R_{n l}^{\mathrm{AE}}(r) / \bar{R}_{l}(r)$ in the region where $r>\tilde{r}_{l}$ and $g_{l}(r)=r^{l+1} f\left(r / r_{l}\right)$. The constants $\delta_{l}$ are adjusted to conserve the norm.

Following the suggestions in the original papers by $\mathrm{D}$. Hamann et al., the position of the outermost maximum, $r_{l}^{\text {Max }}$, of the radial wave-function is used to choose convenient cutoff radii. In APE, $\tilde{r}_{l}$ is adjusted, by default, to be $0.6 r_{l}^{\mathrm{Max}}$ if there are core states present with the same angular momentum and $0.4 r_{l}^{\mathrm{Max}}$ otherwise.

\subsubsection{Troullier-Martins potential}

A different method for the construction of the pseudo wavefunctions was proposed by Troullier and Martins [2,27], based on earlier work by Kerker [28]. This method is much simpler than Hamann's and emphasizes the desired smoothness of the pseudo-potential (although it introduces additional constraints to obtain it). It achieves softer pseudo-potentials for the $2 p$ valence states of the first row and for the $d$ valence states of the transition metals. For other elements both methods produce equivalent potentials.

The pseudo wave-functions are defined as

$R_{l}^{\mathrm{PP}}(r)= \begin{cases}R_{n l}^{\mathrm{AE}}(r), & \text { if } r>r_{l}, \\ r^{l} \mathrm{e}^{p(r)}, & \text { if } r<r_{l},\end{cases}$

with

$p(r)=c_{0}+c_{2} r^{2}+c_{4} r^{4}+c_{6} r^{6}+c_{8} r^{8}+c_{10} r^{10}+c_{12} r^{12}$.

The screened pseudo-potential is obtained by inserting the radial pseudo wave-function (26) into Eq. (13):

$v_{l}^{\mathrm{PP}, \operatorname{scr}}(r)= \begin{cases}v_{n l}^{\mathrm{AE}}(r), & \text { if } r>r_{l}, \\ \varepsilon_{n l}+\frac{l+1}{r} p^{\prime}(r)+\frac{p^{\prime \prime}(r)+\left[p^{\prime}(r)\right]^{2}}{2}, & \text { if } r<r_{l} .\end{cases}$

The coefficients of $p(r)$ are adjusted imposing normconservation, the continuity of the pseudo wave-functions and of their first four derivatives at $r=r_{l}$, and that the screened pseudo-potential has null curvature at the origin. This last condition implies that

$c_{2}^{2}+c_{4}(2 l+5)=0$, 

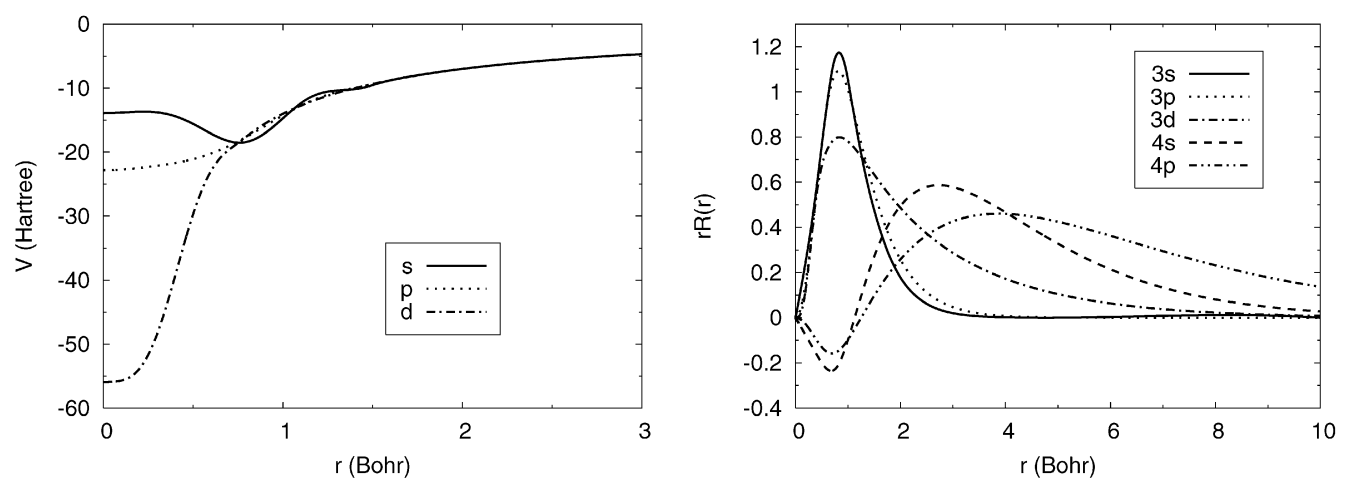

Fig. 1. Chromium pseudo-potentials and pseudo wave-functions generated using the MRPP scheme. The following cutoff radii were used: $r_{c, s}^{\mathrm{sc}}=1.61, r_{c, s}^{\mathrm{v}}=2.80$, $r_{c, p}^{\mathrm{sc}}=1.70, r_{c, p}^{\mathrm{v}}=3.70$, and $r_{c, d}^{\mathrm{v}}=0.80$. All distances are in Bohr. $r_{c}^{\mathrm{sc}}$ denotes the cutoff radii of the semi-core states and $r_{c}^{\mathrm{v}}$ the cutoff radii of the valence states.

and is at the origin of the enhanced smoothness of the Troullier and Martins pseudo-potentials. The resulting set of seven equations includes two non-linear equations, and is solved using the derivative-free Hybrid algorithm provided by the GSL.

\subsubsection{Troullier-Martins potential with explicit incorporation of semi-core states}

The Troullier-Martins scheme presented in the previous section usually allows the generation of very accurate pseudopotentials for most elements. Nevertheless there are cases where the separation between valence and inner core electrons is not well verified, i.e., some core states are not strongly bound or have a large extension. Those core states are known as semicore states. Because of them the TM pseudo-potentials may fail to predict accurately some physical properties like the bond lengths of several $3 d$ transition metal clusters. Reis et al. [8] suggested an extension of the TM scheme to explicitly include the semi-core states into the valence space. This scheme is known as multi-reference pseudo-potentials (MRPP).

Usually it is not necessary to include semi-core states for all the values of the angular momentum $l$. The pseudo-potentials for those values of $l$ should be generated by applying the usual TM scheme.

In the MRPP scheme both the nodeless pseudo wavefunctions for the semi-core states and the single-node pseudo wave-functions for the valence states are required to match the all-electron wave-functions beyond a chosen cutoff radius. This cutoff radius does not need to be the same for the semi-core and the valence states.

As in the normal TM scheme, the pseudo wave-functions for the semi-core states are defined as in Eq. (26) but $p(r)$ is a polynomial of order 9 in $r^{2}$ :

$$
\begin{aligned}
p(r)= & c_{0}+c_{2} r^{2}+c_{4} r^{4}+c_{6} r^{6}+c_{8} r^{8}+c_{10} r^{10} \\
& +c_{12} r^{12}+c_{14} r^{14}+c_{16} r^{16}+c_{18} r^{18} .
\end{aligned}
$$

In order to adjust seven of the ten coefficients of $p(r)$ the same conditions as the ones used for the normal TM scheme are applied to the semi-core pseudo wave-functions. The conditions needed to adjust the three remaining coefficients are applied to the valence pseudo wave-functions. Those conditions are the continuity of the pseudo wave-functions, norm-conservation, and the requirement that the eigenvalues of the valence pseudostates are the same as the all-electron ones.

Because of these extra conditions this set of non-linear equations is difficult to solve numerically and a careful choice of the cutoff radii is essential to obtain a solution. Typically one should choose the cutoff radii for the semi-core states to be greater than the outermost maximum of the all-electron wavefunction.

We have observed that the numerical solution of the set of non-linear equations is more stable if instead of imposing the condition on the eigenvalue of the valence pseudo-states one uses the continuity of the first derivative of the valence pseudo wave-functions. The reason for this is that the value of the first derivative of the pseudo wave-functions at the cut-off radius is more sensitive to changes in the values of the coefficients than the eigenvalues. It is also computationally less demanding to determine the derivative at a certain radius than to determine the eigenvalue. Note that both conditions are perfectly equivalent.

In Fig. 1 we plot pseudo wave-functions and pseudopotentials for chromium. Those were generated using the MRPP scheme starting from scalar-relativistic all-electron calculations. Semi-core states were included for the $s$ and $p$ components. The $d$ component was generated using the normal TM scheme.

\subsubsection{Relativistic extension of the Troullier-Martins potential}

In the normal TM scheme the pseudo-potential is obtained by inverting a radial Schrödinger-like Kohn-Sham equation (Eq. (13)). When the radial all-electron wave-functions are solutions of the radial Dirac-Kohn-Sham equations (9) the usual procedure is to completely neglect the small component $g(r)$ and to use the large component $f(r)$ as if it were a nonrelativistic wave-function. Even if this procedure is justifiable, it introduces inconsistencies. Indeed the pseudo wave-functions obtained this way are not eigenstates of the Dirac-Kohn-Sham equations for the pseudo-potential. Also, completely neglecting the small component implies that its norm contribution has to be absorbed by the large component. This leads to an error during the unscreening procedure. 

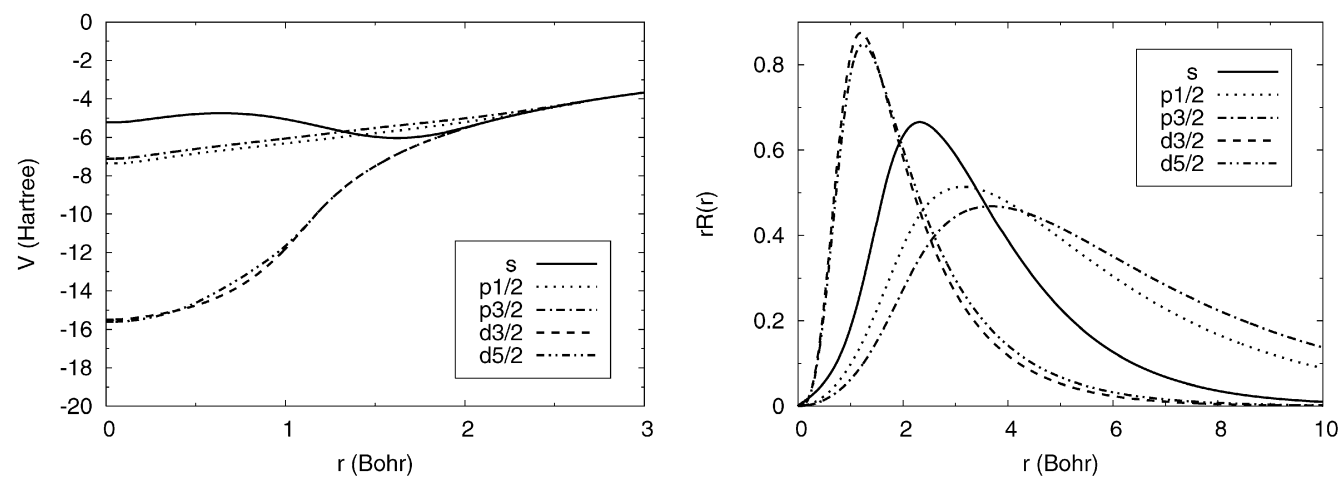

Fig. 2. Gold pseudo-potential and pseudo wave-functions generated using the relativistic extension of the TM scheme. The following cutoff radii were used: $r_{c, s}=2.20, r_{c, p}=3.00$, and $r_{c, d}=1.20$. All distances are in Bohr.

Engel et al. [9] introduced an extension of the TM scheme (RTM) to solve these inconsistencies. In their scheme the two components of the pseudo wave-functions are defined as:

$f_{l j}^{\mathrm{PP}}(r)= \begin{cases}f_{n l j}^{\mathrm{AE}}(r), & \text { for } r>r_{l j}, \\ r^{l} \mathrm{e}^{p(r)}, & \text { for } r<r_{l j},\end{cases}$

$g_{l j}^{\mathrm{PP}}(r)= \begin{cases}g_{n l j}^{\mathrm{AE}}(r), & \text { for } r>r_{l j}, \\ \frac{c\left[(l+1+k) / r+p^{\prime}(r)\right] f^{\mathrm{PP}}(r)}{2 c^{2}-v^{\mathrm{PP}}+\varepsilon_{n l j}}, & \text { for } r<r_{l j} .\end{cases}$

The screened pseudo-potential is in turn given by:

$v_{l j}^{\mathrm{PP}, \operatorname{scr}}(r)= \begin{cases}v_{l j}^{\mathrm{AE}}(r), & \text { for } r>r_{l j}, \\ v_{l j}^{\text {scr,nr }}(r)+\delta v_{l j}(r), & \text { for } r<r_{l j} .\end{cases}$

$v_{l j}^{\text {scr,nr }}(r)$ is the pseudo-potential resulting from the inversion of Eq. (13) and is the same as for the normal TM scheme (see Eq. (28)). $\delta v_{l j}(r)$ is the following relativistic correction:

$\delta v_{l j}=\frac{\left(v_{l j}^{\mathrm{scr}, \mathrm{nr}}-\varepsilon_{n l j}\right)^{2}}{2 c^{2}}+\frac{\left(v_{l j}^{\mathrm{scr}, \mathrm{nr}}\right)^{\prime}}{4 c^{2}}\left(\frac{\left(f_{l j}^{\mathrm{PP}}\right)^{\prime}}{f_{l j}^{\mathrm{PP}}}+\frac{k+1}{r}\right)$.

Finally, the conditions to determine the coefficients of $p(r)$ make use of the Dirac-Kohn-Sham equations.

In Fig. 2 we plot pseudo wave-functions and pseudopotentials for gold. Those were generated using the scheme presented in this section.

\subsection{Non-linear core corrections}

In Eq. (14) a linear dependence of the potential on the density was assumed. Unfortunately, that assumption is only valid for the Hartree contribution and not for the exchangecorrelation part

$v_{\mathrm{xc}}\left[n^{\mathrm{AE}}\right](r) \neq v_{\mathrm{xc}}\left[n^{\text {core }}\right](r)+v_{\mathrm{xc}}\left[n^{\mathrm{PP}}\right](r)$.

For many systems this is not a problem and the linearization of the dependence is an adequate approximation. For other systems though, like elements with few valence electrons or with core orbitals extending into the tail of the valence density, it may be necessary to use a nonlinear core-valence exchangecorrelation scheme. In the scheme suggested by Louie et al. [29] the unscreened pseudo-potential is redefined as:

$v^{\mathrm{PP}}(r)=v^{\mathrm{PP}, \text { scr }}(r)-v_{\text {Hartree }}\left[n^{\mathrm{PP}}\right](r)-v_{\mathrm{xc}}\left[\tilde{n}^{\text {core }}+n^{\mathrm{PP}}\right](r)$,

where a partial core density $\tilde{n}^{\text {core }}$ is used instead of the core density in order to avoid spoiling the smoothness of the potential. This partial core density is built by replacing the core density inside some radius $r_{n l c}$ by an arbitrary function. In APE it is possible to build this arbitrary function by using a sixth order polynomial as suggested by Fuchs et al. [30] or by using the method used in the Martins pseudo-potential code [24].

\subsection{Kleinman and Bylander form of the pseudo-potential}

The semi-local form (Eq. (19)) of the pseudo-potential operator leads to a complicated evaluation of its action on a wavefunction. However the semi-local form can be rewritten in a form that alleviates this computational burden by separating the long and short range components. The long range component is local, and corresponds to the Coulomb tail. Choosing an arbitrary angular momentum component (usually the most repulsive one) and defining

$\delta v_{l}^{\mathrm{PP}}=v_{l}^{\mathrm{PP}}-v_{\text {local }}$,

the pseudo-potential operator can be written as

$\hat{v}_{\mathrm{SL}}=v_{\text {local }}+\sum_{l m}\left|Y_{l m}\right\rangle \delta v_{l}^{\mathrm{PP}}\left\langle Y_{l m}\right|$.

Kleinman and Bylander [31] suggested that the non-local part of Eq. (38) were written as a separable potential, thus transforming the semi-local potential into a truly non-local pseudopotential. If $\varphi_{l m}^{\mathrm{PP}}(\vec{r})=R_{l}^{\mathrm{PP}}(r) Y_{l m}(\hat{r})$ denotes the pseudo wavefunctions obtained with the semi-local pseudo-potential, the Kleinman and Bylander $(\mathrm{KB})$ form is given by

$\hat{v}_{\mathrm{KB}}=v_{\text {local }}+\sum_{l m} \frac{\left|\varphi_{l m}^{\mathrm{PP}} \delta v_{l}^{\mathrm{PP}}\right\rangle\left\langle\varphi_{l m}^{\mathrm{PP}} \delta v_{l}^{\mathrm{PP}}\right|}{\left\langle\varphi_{l m}^{\mathrm{PP}}\left|\delta v_{l}^{\mathrm{PP}}\right| \varphi_{l m}^{\mathrm{PP}}\right\rangle}$.

Using the KB form the action of the potential on a wavefunction becomes much easier to evaluate.

The KB separable form has, however, some disadvantages, leading sometimes to solutions with nodal surfaces that are 
lower in energy than solutions with no nodes [32,33]. These (ghost) states are an artifact of the KB procedure. To eliminate them one can use a different component of the pseudo-potential as the local part of the KB form or choose a different set of core radii for the pseudo-potential generation. As a rule of thumb, the local component of the KB form should be the most repulsive pseudo-potential component.

It is possible to generalize the $\mathrm{KB}$ form to deal with fullyrelativistic $j$-dependent pseudo-potentials $v_{j l}^{\mathrm{PP}}(\vec{r})$ [34]. In this case the $\mathrm{KB}$ form is simply given by:

$\hat{v}_{\mathrm{KB}}=v_{\text {local }}+\sum_{l j m_{j}} \frac{\left|\varphi_{l j m_{j}}^{\mathrm{PP}} \delta v_{l j}^{\mathrm{PP}}\right\rangle\left\langle\varphi_{l j m_{j}}^{\mathrm{PP}} \delta v_{l j}^{\mathrm{PP}}\right|}{\left\langle\varphi_{l j m_{j}}^{\mathrm{PP}}\left|\delta v_{l j}^{\mathrm{PP}}\right| \varphi_{l j m_{j}}^{\mathrm{PP}}\right\rangle}$.

Note that in this case the angular part of the pseudo wavefunctions $\varphi_{l j m_{j}}^{\mathrm{PP}}$ are spherical spinors. This implies that the action of this potential on a wave-function, which should also be a complex spinor, is more complicated to evaluate than for a non-relativistic potential.

\subsection{Pseudo-potential transferability}

The pseudo-potential is able, by construction, to fully reproduce the valence eigenvalues and wave-functions (beyond the core radii) of a single isolated atom. However, when this same atom is embedded in a more complex environment, its wave-functions and eigenvalues differ from those of the isolated atom and one needs to assure that the pseudo-potential will be able to mimic the effect of the true potential in the new chemical environment. In other words, a test of the pseudo-potential transferability is required.

Several transferability tests can be performed, the most obvious one being the comparison of all-electron and pseudopotential results in diverse chemical environments. This test can be preceded by a series of simpler tests on the isolated atom pseudo wave-functions and eigenvalues that immediately identify ill-constructed pseudo-potentials. Nevertheless, even if the pseudo-potential passes all these simpler tests, a true assessment of its transferability still requires a comparison of pseudo-potential and all-electron results in different chemical environments.

One way to force the pseudo-potential to act on a given electron in the same way as the true potential is to require it to have the same scattering phase shifts as the all-electron potential. The (energy-dependent) phase shifts at some distance $r$ from the atom can be shown to depend only on the logarithmic derivative of the wave-function at that distance (and, obviously, on the energy). The norm-conserving pseudo-potentials are constructed in a way that ensures that, for any $r \geqslant r_{l}$ (Eq. (18)), the logarithmic derivatives of the pseudo and true wave-functions coincide. But this is true only for the reference energy $\varepsilon_{l}$ and the eigenvalues depend on the chemical environment. A supplementary condition must then be introduced: the energydependence of the logarithmic derivatives (for $r \geqslant r_{l}$ ) should be the same in the pseudo-potential and all-electron atoms. This constraint can be alleviated by restricting it to energies close to the reference energy or, in other words, by requiring only that the first energy derivatives of the logarithmic derivatives coincide. This ensures that the pseudo-potential will reproduce the changes in the eigenvalues due to small changes in the self-consistent potential. This condition seems daunting, but a closer look at the norm-conservation constraint (Eq. (16)) reveals that [35]

$$
\frac{\partial}{\partial \varepsilon_{l}} \frac{\mathrm{d}}{\mathrm{d} r} \ln R_{l}^{\mathrm{PP}}(r)=-\frac{2}{\left|R_{l}^{\mathrm{PP}}(r)\right|^{2} r^{2}} \int_{0}^{r} \mathrm{~d} r^{\prime}\left|R_{l}^{\mathrm{PP}}\left(r^{\prime}\right)\right|^{2} r^{\prime 2},
$$

which indicates that, for any $r \geqslant r_{l}$, the energy derivatives of the pseudo and true logarithmic derivatives should coincide. In fact, it is not necessary to impose this supplementary condition, since norm-conservation ensures, as a bonus, that the condition is always satisfied. One should simply check that the condition is indeed obeyed. In APE this check is done by computing the logarithmic derivatives of the wave-functions as a function of the eigenvalue for both the all-electron potential and the pseudo-potential at some diagnostic radius outside the core region. A suitable choice for this radius is half the typical inter-atomic distance. The range of eigenvalues to be considered should be the one where the valence states are expected to form molecular orbitals or Bloch bands.

Another easy test of transferability involves the comparison of excitation energies in the pseudo-atom and in the all-electron atom. Due to the implicit inclusion of the reference configuration core states in the pseudo-potential, this comparison should be performed keeping the all-electron core states frozen. This methodology can be extended to the analysis of the change in valence eigenvalues induced by a variation in valence orbital occupancies, giving information on the accuracy of the description of the chemical hardness.

As mentioned above, the transformation of the pseudopotentials to the Kleinman and Bylander form can introduce unphysical states. If this form is to be used, further transferability tests are required, namely the inspection of the bound state spectrum in order to rule out the existence of such states. This analysis is done automatically by APE using the method proposed by Gonze et al. [33] whenever the KB projectors are computed.

If a pseudo-potential is found to be poorly transferable, the most obvious method to render it more transferable involves at least one of these modifications in its construction procedure: changing the core radii, including more valence states, incorporating non-local core corrections, or including different angular momentum projectors.

\section{Program structure}

In order to make the compilation and installation of the code user friendly APE uses the GNU autotools. This means that, on most machines, issuing the following commands:

./configure

make

make install 


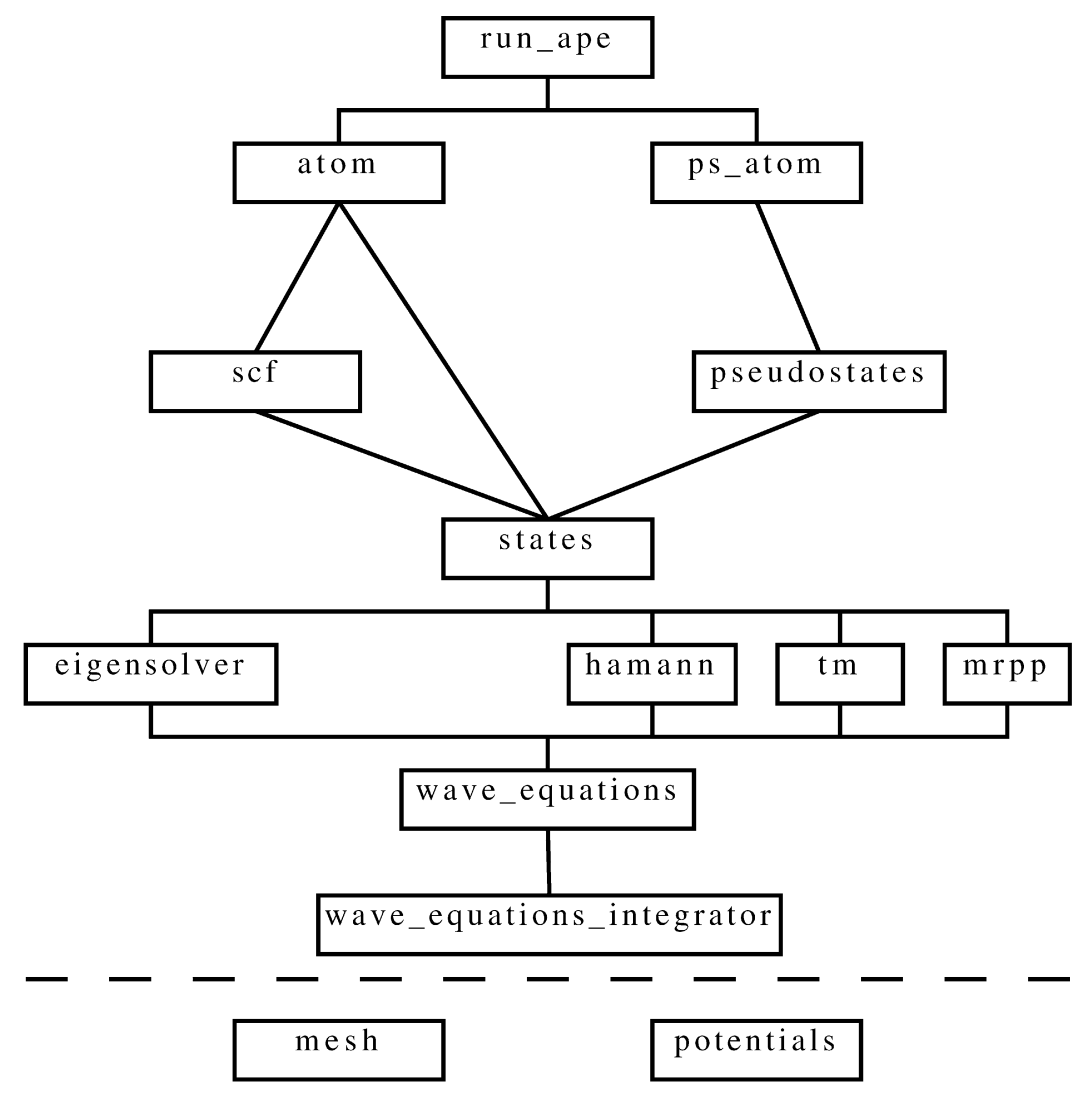

Fig. 3. Simplified schematic chart of the program. Here are depicted the relationships between the most important modules. The modules below the dashed line are used by most of the other modules.

will compile and install the code, as long as there are $\mathrm{C}$ and Fortran 90/95 compilers available and the GSL library is installed in some standard directory. More information about compilation and installation can be found in the code manual.

For the program structure an effort was made to use, whenever possible, object-oriented programming concepts and solutions. The basic structure of almost all the modules is the same: one data structure followed by routines to initialize, to use and to end that structure.

In Fig. 3 it is possible to see how the most important modules are connected to each other. Here follows a brief description of their contents:

- run_ape: executes the necessary tasks for each run mode.

- ps_atom: contains the routines to deal with the pseudoatom (the pseudo-states and the corresponding pseudopotentials).

- atom: performs operations on the set of atomic orbitals, like computing the total energy or the atomic density.

- scf: performs the self-consistent cycle procedure in order to obtain the ground-state of the system from the Kohn-Sham equations.

- pseudostates: a pseudo-state includes all the information necessary to generate a given pseudo-potential component.

- states: this module contains routines to deal with orbitals. An orbital is defined as a set of quantum numbers, an energy and the corresponding wave-function.
- eigensolver: given a potential and a set of quantum numbers, it finds the eigenvalue and eigenstate.

- hamann: given an all-electron potential and wave-functions, generates the corresponding pseudo-potentials using the Hamann scheme.

- tm: given an all-electron potential and wave-functions, generates the corresponding pseudo-potentials using the Troullier-Martins scheme or its relativistic extension.

- mrpp: given an all-electron potential and wave-functions, generates the corresponding pseudo-potentials using the Troullier-Martins scheme with explicit incorporation of semi-core states.

- wave_equation: for a given set of quantum numbers, an energy, and a potential it returns the wave-function or the logarithmic derivative of the wave-function at a given point.

- wave_equation_integrator: performs the integration of the ordinary differential equations.

- potentials: handles the all-electron potentials and pseudopotentials.

- mesh: defines the mesh date type and contains the routines to generate the grids.

The input files are read using a parser that was originally written for the Octopus project [20]. A the beginning of a run, the parser reads the input file, parses it, and generates a list of variables. The list is then available to the other parts of the code. There are two types of variables: scalars and blocks. A scalar variable is defined by: 
$\operatorname{var}=\exp$

var is the name of the variable and exp is the value that should be assigned to the variable. Blocks are collections of values arranged in row and column format:

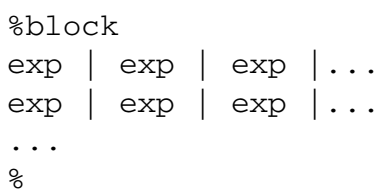

More information about the parser and the input variables can be found in the code manual.

Besides the code itself the APE package includes some useful extra bits in the following sub-directories:

- doc: A comprehensive manual with a complete description of the input file variables.

- sample: Input files to be used as examples.

- testsuite: A set of tests to verify the compilation of the code and to validate changes introduced in the code.

\section{Acknowledgements}

We would like to thank M.A.L. Marques, S. Botti, L. Matos, and M. Gruening for useful discussing and beta-testing. This work was supported by Fundação para a Ciência e Tecnologia through grant \#POCI/FIS/58309/2004. M.J.T.O. thankfully acknowledges financial support from the Portuguese FCT (contract \#SFRH/BD/12712/2003).

\section{Appendix A. Input and selected output files for Silicon}

Below we present an input file for the generation of a Silicon pseudo-potential. This potential was generated by firstly solving the all-electron non-relativistic equation (Eq. (4)) using the local density approximation for exchange and correlation. The pseudo-potential was created in the same run, as CalculationMode has been set to ae + pp.

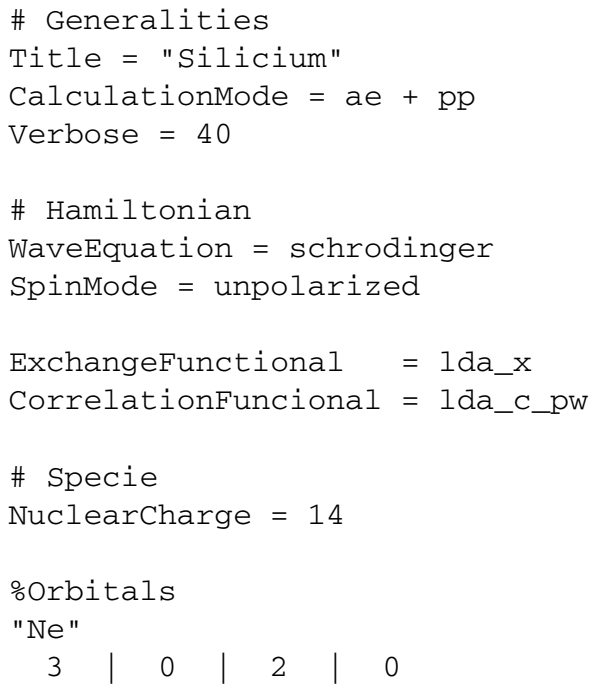

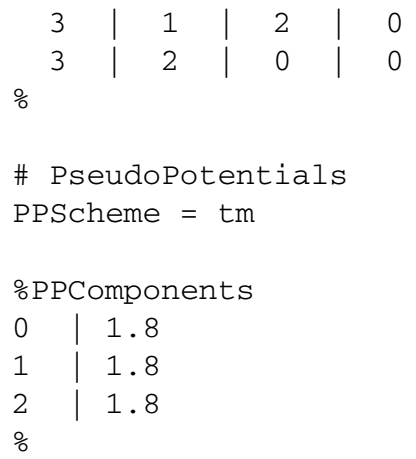

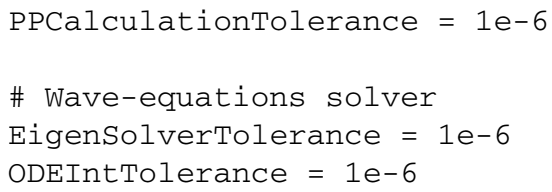

The result of the all-electron calculation outlined above is the set of 5 occupied orbitals plus an unoccupied $3 d$ orbital. All the orbitals are saved in the files ae/wf-*. The file ae/info contains the information printed below.

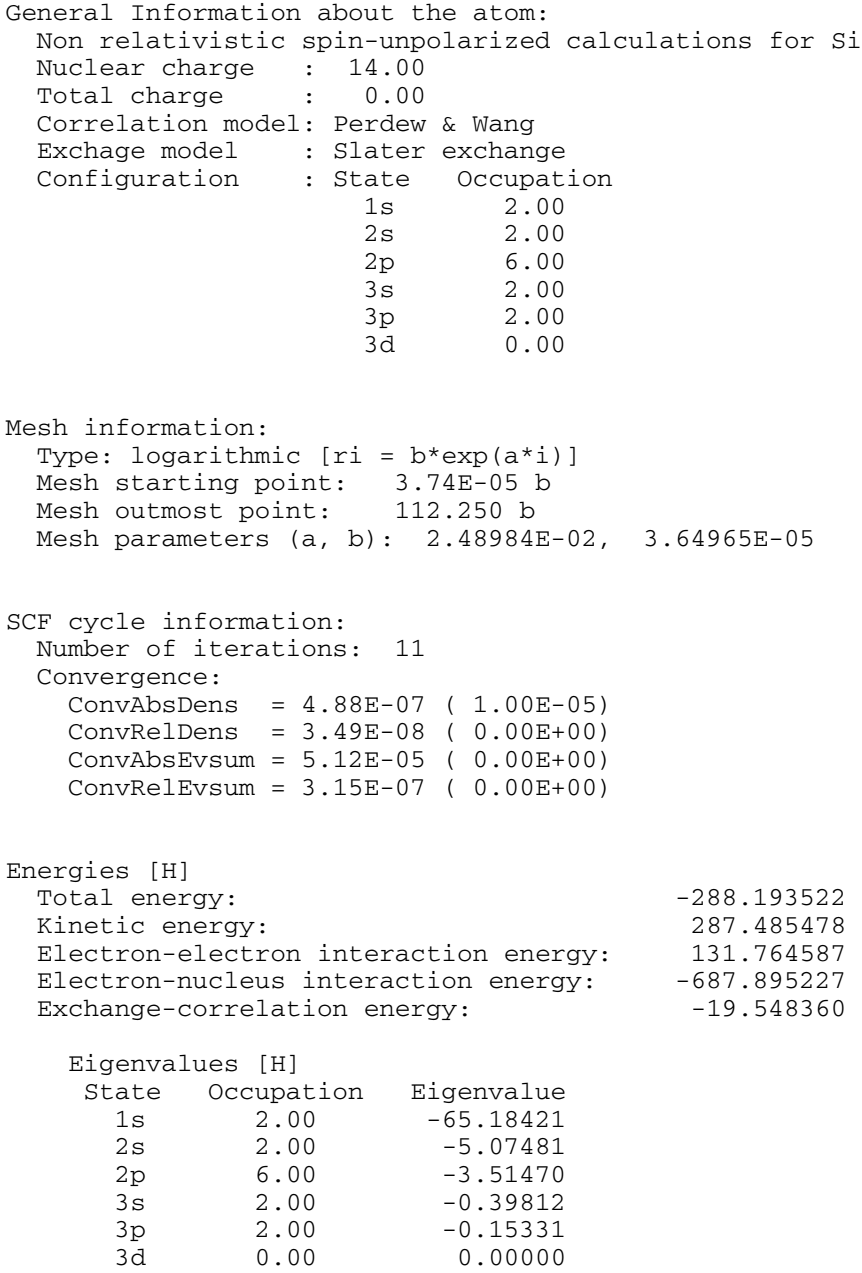

At the end of the all-electron calculation, APE automatically generated a Troullier-Martins pseudo-potential. The contents of $\mathrm{pp} /$ info are printed below. The pseudo wave-functions 
were stored in the files $\mathrm{pp} / \mathrm{wf}-^{*}$, while the files $\mathrm{pp} / \mathrm{pp}-$ * contain the associated pseudo-potential components. The pseudo-potential operator is saved in the file Si.UPF, in the default output format (PWscf).

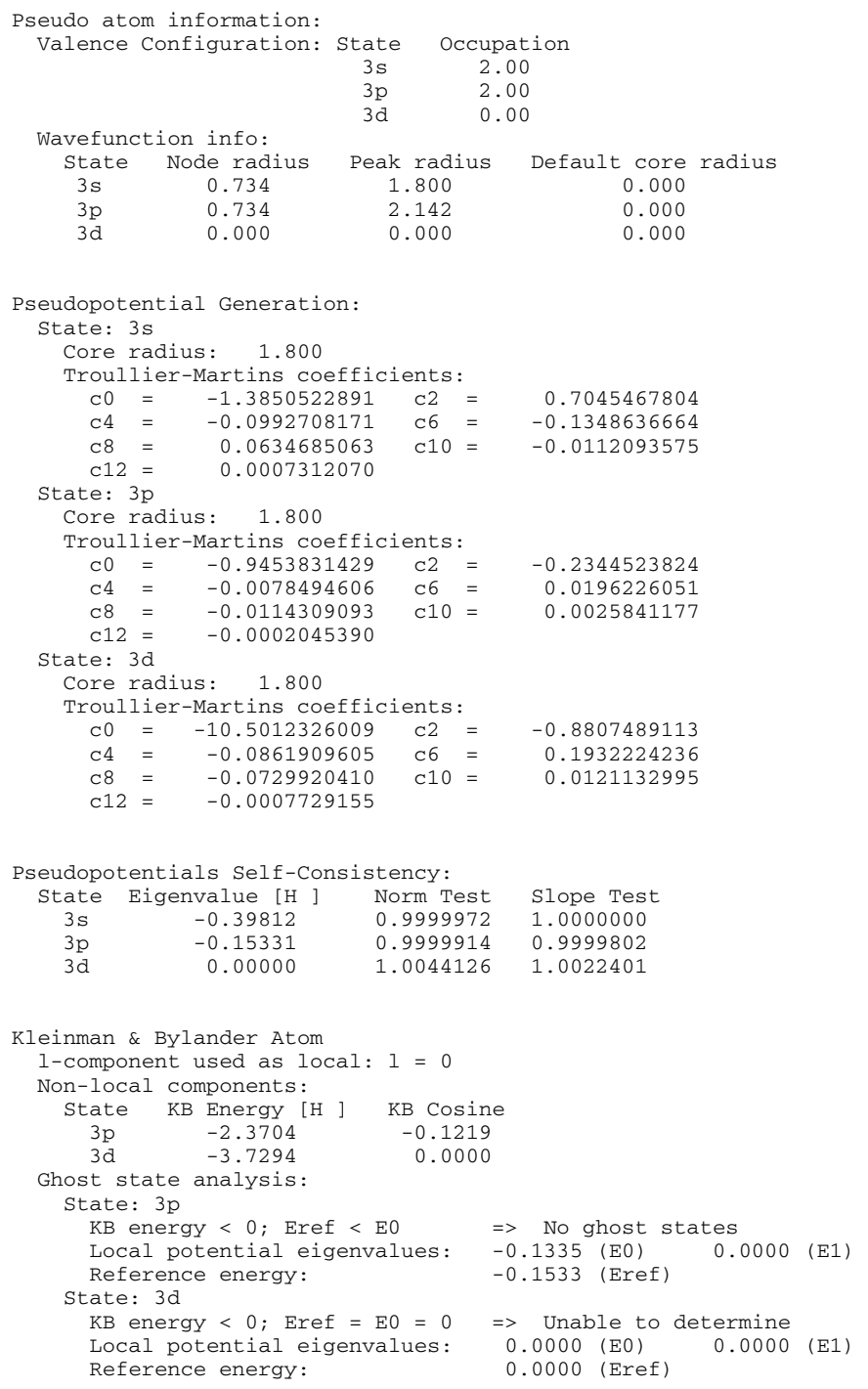

\section{References}

[1] C. Fiolhais, F. Nogueira, M. Marques (Eds.), A Primer in Density Functional Theory, Lectures Notes in Physics, vol. 620, Springer, Berlin, 2003; R.M. Dreizler, E.K.U. Gross, Density Functional Theory, Springer, Berlin, 1990;
R.G. Parr, W. Yang, Density Functional Theory of Atoms and Molecules, Oxford University Press, New York, 1989.

[2] N. Troullier, J.L. Martins, Phys. Rev. B 43 (1991) 1993.

[3] D.R. Hamann, Phys. Rev. B 40 (1989) 2980.

[4] S. Goedecker, M. Teter, J. Hutter, Phys. Rev. B 54 (1996) 1703.

[5] C. Hartwigsen, S. Goedecker, J. Hutter, Phys. Rev. B 58 (1998) 3641.

[6] A.M. Rappe, K.M. Rabe, E. Kaxiras, J.D. Joannopoulos, Phys. Rev. B 41 (1990) 1227.

[7] D. Vanderbilt, Phys. Rev. B 41 (1990) 7892.

[8] C.L. Reis, J.M. Pacheco, J.L. Martins, Phys. Rev. B 68 (2003) 155111.

[9] E. Engel, H. Höck, S. Varga, Phys. Rev. B 63 (2001) 125121.

[10] J.M. Soler, E. Artacho, J.D. Gale, A. García, J. Junquera, P. Ordejón, D. Sánchez-Portal, J. Phys.: Condens. Matter 14 (2002) 2745.

[11] M. Fuchs, M. Scheffler, Comput. Phys. Comm. 119 (1999) 67.

[12] X. Gonze, J.M. Beuken, R. Caracas, F. Detraux, M. Fuchs, G.M. Rignanese, L. Sindic, M. Verstraete, G. Zerah, F. Jollet, M. Torrent, A. Roy, M. Mikami, Ph. Ghosez, J.Y. Raty, D.C. Allan, Comput. Mater. Sci. 25 (2002) 478

[13] S. Baroni, A. Dal Corso, S. de Gironcoli, P. Giannozzi, C. Cavazzoni, G. Ballabio, S. Scandolo, G. Chiarotti, P. Focher, A. Pasquarello, K. Laasonen, A. Trave, R. Car, N. Marzari, A. Kokalj, http://www.pwscf.org/.

[14] P. Hohenberg, W. Kohn, Phys. Rev. B 136 (1964) 864; W. Kohn, L.J. Sham, Phys. Rev. A 140 (1965) 1133.

[15] U. von Barth, L. Hedin, J. Phys. C 5 (1972) 1629.

[16] D.D. Koelling, B.N. Harmon, J. Phys. C 10 (1977) 3107.

[17] R.M. Dreizler, A Primer in Density Functional Theory, in: C. Fiolhais, F. Nogueira, M. Marques (Eds.), Springer, Berlin, 2003 (Chapter 3).

[18] M. Galassi, et al., GNU Scientific Library Reference Manual, second ed., ISBN 0954161734, http://www.gnu.org/software/gsl/.

[19] R.P. Brent, Algorithms for Minimization without Derivatives, PrenticeHall, Englewood Cliffs, NJ, 1973 (Chapter 4).

[20] M.A.L. Marques, A. Castro, G.F. Bertsch, A. Rubio, Comput. Phys. Comm. 151 (2003) 60;

A. Castro, H. Appel, M. Oliveira, C.A. Rozzi, X. Andrade, F. Lorenzen, M.A.L. Marques, E.K.U. Gross, A. Rubio, Phys. Stat. Sol. B 243 (2006) 2465, http://www.tddft.org/programs/octopus/.

[21] D.D. Johnson, Phys. Rev. B 38 (1988) 12807.

[22] W.C. Topp, J.J. Hopfield, Phys. Rev. B 7 (1973) 1295.

[23] S.C. Watson, E.A. Carter, Phys. Rev. B 58 (1998) R13309; V. Cocula, F. Starrost, S.C. Watson, E.A. Carter, J. Chem. Phys. 119 (2003) 7659 .

[24] http://bohr.inesc-mn.pt/ jlm/pseudo.html.

[25] D.R. Hamann, M. Schlüter, C. Chiang, Phys. Rev. Lett. 43 (1979) 1494.

[26] G.B. Bachelet, D.R. Hamann, M. Schlüter, Phys. Rev. B 26 (1982) 4199.

[27] N. Troullier, J.L. Martins, Solid State Commun. 74 (1990) 613.

[28] G.P. Kerker, J. Phys. C: Solid State Phys. 13 (1980) L189.

[29] S.G. Louie, S. Froyen, M.L. Cohen, Phys. Rev. B 26 (1982) 1738.

[30] M. Fuchs, M. Bockstedte, E. Pehlke, M. Scheffler, Phys. Rev. B 57 (1998) 2134.

[31] L. Kleinman, D.M. Bylander, Phys. Rev. Lett. 48 (1982) 1425.

[32] X. Gonze, P. Käckell, M. Scheffler, Phys. Rev. B 41 (1990) 12264.

[33] X. Gonze, R. Stumpf, M. Scheffler, Phys. Rev. B 44 (1991) 8503.

[34] G. Theurich, N.A. Hill, Phys. Rev. B 64 (2001) 073106.

[35] E.L. Shirley, D.C. Allan, R.M. Martin, J.D. Joannopoulos, Phys. Rev. B 40 (1989) 3652. 